\title{
Nobeyama 45m CO Galactic Plane Survey: Filament properties and star formation in M17
}

\author{
Atsushi Nishimura ${ }^{1}$, Tomofumi Umemoto ${ }^{1}$, Tetsuhiro Minamidani ${ }^{1}$, \\ Nario Kuno ${ }^{2,3}$, Tomoka Tosaki ${ }^{4}$, Shinji Fujita ${ }^{2}$, Mitsuhiro Matsuo ${ }^{5}$, \\ Yuya Tsuda ${ }^{6}$, Satoshi Ohashi ${ }^{7}$ and NRO-GPS team \\ ${ }^{1}$ Nobeyama Radio Observatory, National Astronomical Observatory of Japan, Minamimaki, \\ Minamisaku, Nagano, Japan \\ ${ }^{2}$ Department of Physics, Graduate School of Pure and Applied Sciences, University of \\ Tsukuba, Tsukuba, Ibaraki, Japan \\ ${ }^{3}$ Center for Integrated Research in Fundamental Science and Engineering (CiRfSE), University \\ of Tsukuba, Tsukuba, Ibaraki, Japan \\ ${ }^{4}$ Department of Geoscience, Joetsu University of Education, Joetsu, Niigata, Japan \\ ${ }^{5}$ Graduate School of Science and Engineering, Kagoshima University, Kagoshima, Kagoshima, \\ Japan \\ ${ }^{6}$ Graduate School of Science and Engineering, Meisei University, Hino, Tokyo, Japan \\ ${ }^{7}$ Department of Astronomy, The University of Tokyo, Bunkyo-ku, Tokyo, Japan \\ email: atsushi.nishimura@nao.ac.jp
}

\begin{abstract}
We present the ${ }^{12} \mathrm{CO}(J=1-0),{ }^{13} \mathrm{CO}(J=1-0)$, and $\mathrm{C}^{18} \mathrm{O}(J=1-0)$ maps of the M17 giant molecular clouds (GMCs) obtained as a part of the Nobeyama 45m CO Galactic Plane Survey. The observations cover the entire area of M17 SW and M17 N clouds at an angular resolution of $\sim 15^{\prime \prime}$ which corresponds to $\sim 0.15 \mathrm{pc}$. We found that the $\mathrm{N}$ cloud consists of a couple of twisted filaments, they are extended in parallel toward the $\mathrm{H}$ II region. The typicall width of the filaments is $\sim 0.5 \mathrm{pc}$ in ${ }^{13} \mathrm{CO}$ intensity map. Most of young stellar objects (YSOs) are located on the filaments which have a bright rim structure in $8 \mu \mathrm{m}$ at the filament edge facing the H II region. Furthermore, the time scale of the YSOs formation on the bright rim is comparable with that of NGC 6618 cluster which provides UV photons for the region. This fact indicates that the cluster triggered to form YSOs in N cloud. We also investigated the geometry of the $\mathrm{H}$ II region and GMCs by comparing spatial distribution of ${ }^{12} \mathrm{CO}$ velocity channel map and infrared dark cloud, and then found that NGC 6618 is possibly formed by the cloud cloud colision.
\end{abstract}

Keywords. ISM: clouds, ISM: individual (M17), radio lines: ISM, stars: formation

\section{Introduction}

Most stars are formed in giant molecular clouds (GMCs) in the Galaxy. Recently, Herschel observations reveal the ubiquitous existence of the filamentary structure (e.g., André et al.2010) and demonstrate that spatially complete high-resolution observations are essential to understand the whole picture of star formation in the GMCs. Molecular line observations have an advantage in GMC studies because it provides velocity information (e.g., Nishimura et al.2015) while its limited sensitivity prevent us to obtaine large scale GMC images with sub-parsec resolution. Nobeyama 45m CO Galactic Plane Survey (Umemoto et al.in prep.) is realized by a newly developped multi-beam receiver FOREST (Minamidani et al.in prep.) and the survey provides the most powerful dataset for this purpose.

M17 is one of the most famous H II region excited by the high-mass star cluster NGC 6618 (the region is reviewed by Chini \& Hoffmeister 2008). The cluster consists of several O stars and estimated to be very young $(\sim 0.5 \mathrm{Myr}$; Hoffmeister et al.2008). The region mainly consists of two GMCs, M17 SW and M17 N (Lada 1976). We observed entire region of the clouds by 

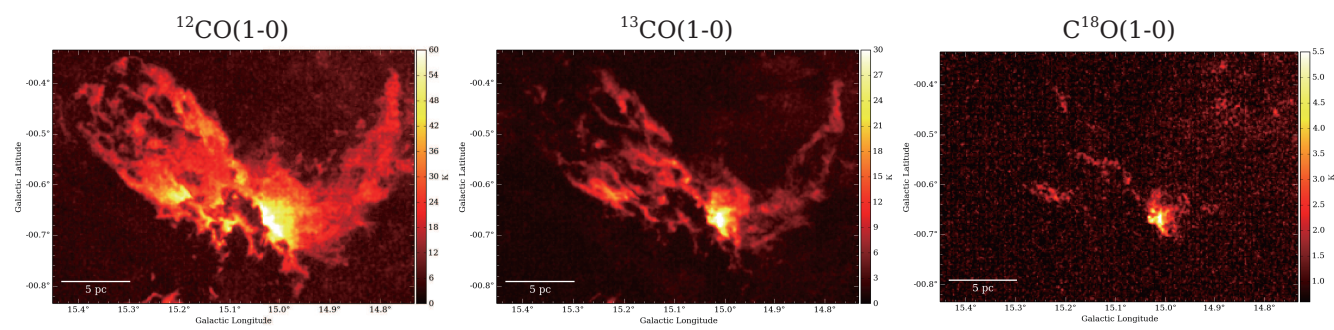

Figure 1. Peak intensity maps of ${ }^{12} \mathrm{CO}(J=1-0),{ }^{13} \mathrm{CO}(J=1-0)$, and $\mathrm{C}^{18} \mathrm{O}(J=1-0)$.

using Nobeyama $45 \mathrm{~m}$ telescope and obtained ${ }^{12} \mathrm{CO}(J=1-0),{ }^{13} \mathrm{CO}(J=1-0)$, and $\mathrm{C}^{18} \mathrm{O}(J=1-0)$ images at an angular resolution of $\sim 15^{\prime \prime}$.

\section{Results and discussions}

Figure 1 shows the peak intensity maps of CO lines observed with Nobeyama $45 \mathrm{~m}$ telescope. The M17 molecular cloud complex is fully covered with improved angular resolution compared with previous ${ }^{12} \mathrm{CO}(J=2-1)$, and ${ }^{13} \mathrm{CO}(J=2-1)$ observation carried out by Povich et al. (2009).

We use DisPerSE algorithm (Sousbie 2011) to identify filamentary structures from the cube data of ${ }^{13} \mathrm{CO}$ emission. After the filtering of daubtful candidates, we found that the typical filament width is $\sim 0.5 \mathrm{pc}$ and the density profiles seem to be follow Plummer-like function for both SW and $\mathrm{N}$ clouds. In the $\mathrm{N}$ cloud, almost all young stellar objects (YSOs) identified by Povich et al.(2013) are located on the filaments. Most of the YSOs are classified as Class 0/I and a few are Class II/III. All filaments associated with YSOs have bright rim features at $8 \mu \mathrm{m}$ with the direction of the cluster indicates that the star formation in $\mathrm{N}$ cloud is triggered by the feedback of the cluster.

We compared ${ }^{12} \mathrm{CO}(J=1-0)$ velocity channell maps with infrared images obtained by Spitzer/ IRAC. We found that $20 \mathrm{~km} \mathrm{~s}^{-1}$ components (blue) are mainly corresponding to dark lane observed in $\mathrm{N}$ cloud, while $23 \mathrm{~km} \mathrm{~s}^{-1}$ components (red) have no dark cloud counterparts. Therefore, $\mathrm{N}$ cloud filaments (blue component) are located at the near side of the $\mathrm{H}$ II region and the red conponent are located at the far side of the $\mathrm{H}$ II region. Furthermore, both blue and red components are seen around the cluster. One possibility which satisfy the results is that the blue and red components were collided and this cloud cloud colision triggered NGC 6618 formation.

\section{References}

André, P., Men'shchikov, A., Bontemps, S., et al. 2010, A\&SA, 518, L102

Chini, R., \& Hoffmeister, V. 2008, Handbook of Star Forming Regions, Volume II, 625

Hoffmeister, V. H., Chini, R., Scheyda, C. M., et al. 2008, ApJ, 686, 310

Lada, C. J. 1976, ApJS, 32, 603

Minamidani, T., Nishimura, A., Miyamoto, Y., et al. in prep.

Nishimura, A., Tokuda, K., Kimura, K., et al. 2015, ApJS, 216, 18

Povich, M. S., Churchwell, E., Bieging, J. H., et al. 2009, ApJ, 696, 1278

Povich, M. S., Kuhn, M. A., Getman, K. V., et al. 2013, ApJS, 209, 31

Sousbie, T. 2011, MNRAS, 414, 350

Umemoto, T., Minamidani, T., Nishimura, A., et al. in prep. 